Pacific Journal of Mathematics

JONES POLYNOMIALS OF PERIODIC LINKS 


\section{JONES POLYNOMIALS OF PERIODIC LINKS}

\section{Kunio Murasugi}

Let $L$ be a link in $S^{3}$ which has a prime period and $L_{*}$ be its factor link. Several relationships between the Jones polynomials of $L$ and $L_{*}$ are proved. As an application, it is shown that some knot cannot have a certain period.

1. Introduction. Let $L$ be an oriented link that has period $r>1$. That is, there exists an orientation preserving auto-homeomorphism $\phi: S^{3} \rightarrow S^{3}$ of order $r$ with a set of fixed points $F \cong S^{1}$ disjoint from $L$ and which maps $L$ onto itself. By the positive solution of Smith Conjecture, $F$ is unknotted. Let $\Sigma^{3}=S^{3} / \phi$ be the quotient space under $\phi$. Since $F$ is unknotted, $\Sigma^{3}$ is again a 3 -sphere, and $S^{3}$ is the $r$-fold cyclic covering space of $\Sigma^{3}$ branched along $F$.

Let $\psi: S^{3} \rightarrow \Sigma^{3}$ be the covering projection. Denote $\psi(L)=L_{*}$, which is called the factor link, and let $V_{L}(t)$ and $V_{L_{*}}(t)$ denote, respectively, the Jones polynomials of $L$ and $L_{*}$.

In this paper, we will prove some relationships between $V_{L}(t)$ and $V_{L_{*}}(t)$ which are analogous to those between their Alexander polynomials [M2]. In fact, we will prove

THEOREM 1. Let $r$ be a prime and L a link that has period $r^{q}, q \geq 1$. Then

$$
V_{L}(t) \equiv\left[V_{L_{*}}(t)\right]^{r^{q}} \bmod \left(r, \xi_{r}(t)\right),
$$

where $\xi_{r}(t)=\sum_{j=0}^{r-1}(-t)^{j}-t^{(r-1) / 2}$.

If $L$ is not split, then we are able to prove a slightly more precise formula.

Let $1 \mathrm{k}(X, Y)$ denote the linking number between two simple closed curves $X$ and $Y$ in $S^{3}$. Then we have

THEOREM 2. Let $r$ be a prime and L a non-split link that has period $r^{q}$, $q \geq 1$. 
(1) If $\operatorname{lk}(L, F) \equiv 1(\bmod 2)$, then

$$
V_{L}(t) \equiv\left[V_{L_{*}}(t)\right]^{r^{q}} \bmod \left(r, \eta_{r}(t)\right),
$$

where $\eta_{r}(t)=\left[\sum_{j=0}^{r-2}(j+1)(-t)^{j}\right]\left(1+t^{r}\right)-t^{r-1}$.

(2) If $\operatorname{lk}(L, F) \equiv 0(\bmod 2)$, then

$$
V_{L}(t) \equiv\left[V_{L_{*}}(t)\right]^{r^{q}} \bmod \left(r, \xi_{r}(t)\right) .
$$

Note that $\eta_{r}(t) \equiv 0 \bmod \left(r, \xi_{r}(t)\right)$. (See Lemma 6 in $\S 3$.) As a simple consequence, we obtain

COROllary 3. Let $\mathbf{b}$ be an $n$-braid and let $V_{\mathbf{b}}(t)$ be the Jones polynomial of the closure $\hat{\mathbf{b}}$ of $\mathbf{b}$. Let $r$ be a prime and $q \geq 1$. Then

$$
V_{\mathbf{b}^{r^{q}}}(t) \equiv\left[V_{\mathbf{b}}(t)\right]^{r^{q}} \bmod \left(r, \xi_{r}(t)\right) \text {. }
$$

Formulas (1.1), (1.2), and (1.3) involve slightly larger ideals than those in the corresponding formulas about the Alexander polynomials [M2]. However, they are the best possible. To see this, consider an $n$-component trivial link $L . L$ has any period $r$ and a factor link $L_{*}$ is also an $n$-component trivial link. Since $V_{L}(t)=V_{L_{*}}(t)=(-1)^{n-1}(\sqrt{t}+1 / \sqrt{t})^{n-1}$, the formula $V_{L}(t) \equiv\left[V_{L_{*}}(t)\right]^{r}(\bmod I)$ holds only if the ideal $I$ contains $\xi_{r}(t)$. We should note that while the Alexander polynomial of a link may vanish, the Jones polynomial of a link never vanishes.

Corollary 3 is also verified for $n=3$ by a direct computation using Theorem $21[\mathbf{J}]$ and Theorem [M2].

These formulas may have more theoretical values than practical values. (See Proposition 7 in $§ 4$.) Nevertheless, we can prove that $10_{105}$ cannot have period 7 (Proposition 10). This solves one of several undecided cases for knots with 10 crossings.

2. Proof of Theorem 1. Since it suffices to prove Theorem 1 for $q=1$, we assume that $L$ has a prime period $r$. In this section, we prove that Theorem 2 implies Theorem 1.

Suppose that $L$ has period $r$ and let $\phi$ be an orientation preserving auto-homeomorphism of $S^{3}$ that maps $L$ onto itself. Suppose that $L$ splits into $k$ components $L_{1}, L_{2}, \ldots, L_{k}$. Then $\phi$ must map a split component not having period $r$ onto another split component not having period $r$. Therefore, split components of $L$ are divided into $h+1$ sets $A_{1}=\left\{L_{1}, \ldots, L_{r}\right\}, A_{2}=\left\{L_{r+1}, \ldots, L_{2 r}\right\}, \ldots, A_{h}=\left\{L_{(h-1) r+1}, \ldots, L_{h r}\right\}$ and $B=\left\{L_{h r+1}, \ldots, L_{k}\right\}$ such that any two links in $A_{t}(i=1,2, \ldots, h)$ are ambient isotopic and a link in $B$ has period $r$. The factor link $L_{*}$, then, has $h+(k-h r)(=k-h(r-1))$ split components. Noting that the factor link of the $r$-split component link $L_{s r+1} \cup \cdots \cup L_{(s+1) r}$ is 
$L_{s r+1}, 0 \leq s \leq h-1$, we have

$$
\begin{aligned}
& \text { (1) } V_{L}(t)=\left[-\left(\sqrt{t}+\frac{1}{\sqrt{t}}\right)\right]^{k-1} \prod_{i=1}^{k} V_{L_{t}}(t), \text { and } \\
& \text { (2) } V_{L_{*}}(t)=\left[-\left(\sqrt{t}+\frac{1}{\sqrt{t}}\right)\right]^{k-h(r-1)-1} \prod_{s=0}^{h-1} V_{L_{s r+1}}(t) \prod_{j=h r+1}^{k} V_{L_{J_{*}}}(t) .
\end{aligned}
$$

Now Theorem 2 implies that for $j=h r+1, \ldots, k, V_{L_{j}}(t) \equiv V_{L_{*}}(t)^{r}$ $\bmod \left(r, \xi_{r}(t)\right)$ and hence

$$
\begin{aligned}
V_{L_{*}}(t)^{r} & =\left[-\left(\sqrt{t}+\frac{1}{\sqrt{t}}\right)\right]^{r[k-h(r-1)-1]} \prod_{s=0}^{h-1} V_{L_{s r+1}}(t)^{r} \prod_{j=h r+1}^{k} V_{L_{j_{*}}}(t)^{r} \\
& \equiv\left[-\left(\sqrt{t}+\frac{1}{\sqrt{t}}\right)\right]^{k-h(r-1)-1} \prod_{i=1}^{k} V_{L_{i}}(t) \quad \bmod \left(r, \xi_{r}(t)\right) .
\end{aligned}
$$

Comparing (2.2) with (2.1) (1), we see that Theorem 1 will follow from Lemma 4 below.

LEMMA 4. For a prime $r$,

$$
\begin{aligned}
&(-1)^{k-h(r-1)-1}\left(\sqrt{t}+\frac{1}{\sqrt{t}}\right)^{k-h(r-1)-1} \\
& \equiv(-1)^{k-1}\left(\sqrt{t}+\frac{1}{\sqrt{t}}\right)^{k-1} \bmod \left(r, \xi_{r}(t)\right) .
\end{aligned}
$$

Proof. Since $\xi_{r}(t) \equiv(1+t)^{r-1}-t^{(r-1) / 2}(\bmod r)$ by Lemma 6 (proved in §3), it follows that $(\sqrt{t}+(1 / \sqrt{t}))^{r-1}=((t+1) / \sqrt{t})^{r-1} \equiv 1$ $\left(\bmod \left(r, \xi_{r}(t)\right)\right)$. Since $r$ is a prime, $(-1)^{k-h(r-1)-1} \equiv(-1)^{k-1}(\bmod r)$.

3. Proof of Theorem 2. We may assume that $q=1$ and $L$ is not split.

Let $\zeta$ be the rotation of $R^{2}$ about the origin 0 through $2 \pi / r$. Since $L$ is a link having period $r, L$ has a diagram $\tilde{L}(\notin\{0\})$ on $R^{2}$ which is divided into $r$ pieces $\tilde{L}_{0}, \tilde{L}_{1}, \ldots, \tilde{L}_{r-1}$ such that $\zeta\left(\tilde{L}_{i}\right)=\tilde{L}_{i+1}, \quad i=$ $0,1, \ldots, r-1, \tilde{L}_{r}=\tilde{L}_{0}$. Let $R(0,2 \pi / r)$ be the closed domain bounded by two half lines $\theta=0$ and $\theta=2 \pi / r$ in the polar coordinate system. We may assume that $\tilde{L}_{0}=\tilde{L} \cap R(0,2 \pi / r)$. Let $A_{1}, A_{2}, \ldots, A_{l}$ be the points of intersection of $\tilde{L}_{0}$ and the line $\theta=0$ and let $\zeta\left(A_{i}\right)=B_{i}, i=1,2, \ldots, l$, $A_{i} \neq B_{i}$. By joining $A_{i}$ and $B_{i}$ on $R^{2}$ by a circle $C_{i}$ centered 0 , we obtain a diagram $\tilde{L}_{*}$ of the factor link $L_{*}=\psi(L)$. For simplicity, we write $\tilde{L}_{*}=\tilde{L} / \zeta . \tilde{L}_{*}$ divides $R^{2}$ into finitely many domains, which we classify as shaded or unshaded. Now unshading the domain containing 0 , we have the graph $\Gamma_{*}$ of $\tilde{L}_{*}$. We may take 0 as one vertex of $\Gamma_{*}$. Furthermore, we 
can assign +1 or -1 to each edge of $\Gamma_{*}$ [M4]. Similarly, we have the graph $\Gamma$ of $\tilde{L}$ by unshading the domain containing $0 . \Gamma$ is also an oriented graph. Using $\Gamma$ and $\Gamma_{*}$, we can evaluate $V_{L}(t)$ and $V_{L_{*}}(t)$ as follows. (See [M4].)

Let $p^{\prime}$ and $n^{\prime}$ be, respectively, the number of positive and negative edges in $\Gamma$. Let $S(a, b), 0 \leq a \leq p^{\prime}$ and $0 \leq b \leq n^{\prime}$, be the collection of subgraphs obtained from $\Gamma$ by removing exactly $a$ positive edges and $b$ negative edges. $S(a, b)$ contains $\left(\begin{array}{c}p^{\prime} \\ a\end{array}\right)\left(\begin{array}{c}n^{\prime} \\ b\end{array}\right)$ subgraphs.

For $\gamma \in S(a, b)$, let $\mu(\gamma)=b_{0}(\gamma)+b_{1}(\gamma)$, where $b_{i}(\gamma), i=0,1$, denotes the $i$ th Betti number of $\gamma$ as a 1-complex. Then the bracket polynomial $P_{\tilde{L}}(A)$ defined in $[\mathbf{K}]$ associated with the link diagram $\tilde{L}$ is given by the following formula:

$$
P_{\tilde{L}}(A)=\sum_{\substack{0 \leq a \leq p^{\prime} \\ 0 \leq b \leq n^{\prime}}} A^{p^{\prime}-2 a-n^{\prime}+2 b} \sum_{\gamma \in S(a, b)}\left[-\left(A^{2}+A^{-2}\right)\right]^{\mu(\gamma)-1} .
$$

Note that (3.1) is equivalent to the formula (2.10) in [M4].

We will use (3.1) to evaluate $P_{\tilde{L}}(A)$ and $P_{\tilde{L}_{*}}(A)$.

Let $p$ and $n$ be, respectively, the number of positive and negative edges in $\Gamma_{*}$. Then $\Gamma$ has exactly $r p$ positive and $r n$ negative edges, i.e. $p^{\prime}=r p$ and $n^{\prime}=r n$. Let $S_{*}(a, b)$ be the collection of subgraphs of $\Gamma_{*}$ which is defined in a similar way to $S(a, b)$. Then we have

$$
P_{\check{L}_{*}}(A)=\sum_{\substack{0 \leq a \leq p \\ 0 \leq b \leq n}} A^{p-2 a-n+2 b} \sum_{\gamma_{*} \in S_{*}(a, b)}\left[-\left(A^{2}+A^{-2}\right)\right]^{\mu\left(\gamma_{*}\right)-1} .
$$

Since the rotation $\zeta: R^{2} \rightarrow R^{2}$ maps $\tilde{L}$ onto itself, we may assume that $\zeta$ maps the graph $\Gamma$ onto itself, preserving the sign of each edge. In other words, $\zeta$ defines an automorphism of the oriented graph $\Gamma$.

If $\operatorname{lk}(L, F) \equiv 1(\bmod 2)$, then the unbounded domain is shaded. Therefore, $\zeta$ fixes only the origin 0 . If $\operatorname{lk}(L, F) \equiv 0(\bmod 2)$, however, the unbounded domain is unshaded, and hence, $\zeta$ keeps exactly two vertices 0 and $\infty$ fixed, where $\infty$ is a point associated with the unbounded domain. Therefore, if $1 \mathrm{k}(L, F) \equiv 0(\bmod 2), \zeta$ may be considered as an automorphism of the graph $\Gamma$ in $S^{2}$ which keeps the north and south poles fixed.

Case A. $1 \mathrm{k}(L, F) \equiv 1(\bmod 2)$.

In this case, $\Gamma$ is the $r$-fold cyclic covering of $\Gamma$ branched at 0 . Take $\gamma \in S(a, b)$.

Case 1. $\gamma$ is not fixed under $\zeta$, i.e. $\zeta(\gamma) \neq \gamma$.

This is, of course, the case when $a \not \equiv 0(\bmod r)$ or $b \not \equiv 0(\bmod r)$. In this case, $\gamma, \zeta(\gamma), \zeta^{2}(\gamma), \ldots, \zeta^{r-1}(\gamma)$ are all distinct, but, since any two of 
these are isomorphic, we have exactly $r$ identical terms in $P_{\tilde{L}}(A)$, and they vanish by reducing modulo $r$.

Case 2. $\gamma$ is fixed under $\zeta$ setwise, i.e. $\zeta(\gamma)=\gamma$.

In this case, $a \equiv b \equiv 0(\bmod r)$. Write $a=r a^{\prime}$ and $b=r b^{\prime}$. Then $\gamma$ defines a unique quotient subgraph $\gamma_{*}(=\gamma / \zeta) \in S_{*}\left(a^{\prime}, b^{\prime}\right)$.

Let $\alpha$ and $\alpha_{*}$, be, respectively, the terms in $P_{\tilde{L}}(A)$ and $P_{\tilde{L}_{*}}(A)$ which are associated with $\gamma$ and $\gamma_{*}$. Since $p^{\prime}=r p$ and $n^{\prime}=r n$, we have

$$
\begin{aligned}
& \alpha=A^{r\left(p-2 a^{\prime}-n+2 b^{\prime}\right)}\left[-\left(A^{2}+A^{-2}\right)\right]^{\mu(\gamma)-1}, \quad \text { and } \\
& \alpha_{*}=A^{p-2 a^{\prime}-n+2 b^{\prime}}\left[-\left(A^{2}+A^{-2}\right)\right]^{\mu\left(\gamma_{*}\right)-1} .
\end{aligned}
$$

We will compare $\mu(\gamma)-1$ and $\mu\left(\gamma_{*}\right)-1$.

If we use the fact that $\gamma$ is the $r$-fold cyclic cover of $\gamma_{*}$, it is not difficult to find some relationship between $b_{1}(\gamma)$ and $b_{1}\left(\gamma_{*}\right)$.

Consider connected components of $\gamma$. Let $D_{0}, D_{1}, \ldots, D_{k}$, $D_{1,1}, \ldots, D_{1, r}, D_{2,1}, \ldots, D_{2, r}, \ldots, D_{m, 1}, \ldots, D_{m, r}$ be connected components of $\gamma$ such that

$$
D_{0} \text { contains the origin }\{0\} \text {, and } \zeta\left(D_{0}\right)=D_{0} \text {, }
$$
$D_{i}(i=1,2, \ldots, k)$ is a component $(\notin\{0\})$ of $\gamma$ such that $\zeta\left(D_{i}\right)=D_{i}$, $\left\{D_{J, 1}, \ldots, D_{j, r}\right\},(j=1,2, \ldots, m)$ is a set of components of $\gamma$ which permutes by $\zeta$

Then connected components of $\gamma_{*}$ consist of the sets: $D_{i}^{\prime}=D_{i} / \zeta$ $(i=0,1,2, \ldots, k)$ and $D_{j, 1}^{\prime}=D_{j, 1}(j=1,2, \ldots, m)$.

We compare $b_{1}\left(D_{i}\right)$ and $b_{1}\left(D_{j, \lambda}\right)$ with $b_{1}\left(D_{i}^{\prime}\right)$ and $b_{1}\left(D_{j, 1}^{\prime}\right)$.

\section{LEMMA 5.}

$$
\begin{aligned}
& b_{1}\left(D_{0}\right)=r b_{1}\left(D_{0}^{\prime}\right), \\
& b_{1}\left(D_{i}\right)-1=r\left\{b_{1}\left(D_{i}^{\prime}\right)-1\right\} \text { for } 1 \leq i \leq k, \\
& b_{1}\left(D_{j, 1}\right)=b_{1}\left(D_{j, \lambda}\right)=b_{1}\left(D_{j, 1}^{\prime}\right) \text { for } 1 \leq j \leq m \text { and } \\
& 1 \leq \lambda \leq r .
\end{aligned}
$$

Proof. (1) Let $d_{0}^{\prime}$ and $e_{0}^{\prime}$, denote, respectively, the number of vertices and edges of $D_{0}^{\prime}$. Then, since $D_{0}$ is the $r$-fold cyclic covering of $D_{0}^{\prime}$ branched at 0 , the number of vertices and edges of $D_{0}$ are given by 
$r\left(d_{0}^{\prime}-1\right)+1$ and $r e_{0}^{\prime}$ respectively. Therefore

$$
\begin{aligned}
1-b_{1}\left(D_{0}\right) & =r\left(d_{0}^{\prime}-1\right)+1-r e_{0}^{\prime}=r\left(d_{0}^{\prime}-e_{0}^{\prime}\right)-r+1 \\
& =r\left(1-b_{1}\left(D_{0}^{\prime}\right)\right)-r+1=1-r b_{1}\left(D_{0}^{\prime}\right),
\end{aligned}
$$

and hence, $b_{1}\left(D_{0}\right)=r b_{1}\left(D_{0}^{\prime}\right)$.

(2) Since $D_{1}$ is the $r$-fold (unbranched) cyclic covering of $D_{0}^{\prime}$, it follows that $\chi\left(D_{i}\right)=r \chi\left(D_{i}^{\prime}\right)$, where $\chi$ denotes the Euler characteristic. Since $\chi\left(D_{i}\right)=1-b_{1}\left(D_{i}\right)$, we have

$$
1-b_{1}\left(D_{i}\right)=r \chi\left(D_{i}^{\prime}\right)=r\left\{1-b_{1}\left(D_{i}^{\prime}\right)\right\}
$$

and hence, $b_{1}\left(D_{\imath}\right)-1=r\left\{b_{1}\left(D_{i}^{\prime}\right)-1\right\}$.

(3) is obvious.

Now we compare $\mu(\gamma)-1$ and $\mu\left(\gamma_{*}\right)-1$. Using Lemma 5, we obtain

$$
\begin{aligned}
\mu(\gamma)-1= & b_{1}(\gamma)+b_{0}(\gamma)-1 \\
= & b_{1}\left(D_{0}\right)+\sum_{i=1}^{k} b_{1}\left(D_{i}\right)+\sum_{j=1}^{m} \sum_{\lambda=1}^{r} b_{1}\left(D_{j, \lambda}\right)+k+1+r m-1 \\
= & r b_{1}\left(D_{0}^{\prime}\right)+\sum_{i=1}^{k}\left\{r b_{1}\left(D_{i}^{\prime}\right)-r+1\right\}+\sum_{j=1}^{m} r b_{1}\left(D_{j, 1}^{\prime}\right)+k+r m \\
= & r\left[b_{1}\left(D_{0}^{\prime}\right)+\sum_{i=1}^{k} b_{1}\left(D_{l}^{\prime}\right)+\sum_{i=1}^{m} b_{1}\left(D_{j, 1}^{\prime}\right)+k+1+m-1\right] \\
& -r k-r m-r k+k+k+r m \\
= & r\left[b_{1}\left(\gamma_{*}\right)+b_{0}\left(\gamma_{*}\right)-1\right]-2 k(r-1) \\
= & r\left\{\mu\left(\gamma_{*}\right)-1\right\}-2 k(r-1) .
\end{aligned}
$$

Using this equality, we have

$$
\alpha \equiv \alpha_{*}^{r} \bmod \left[\left\{-\left(A^{2}+A^{-2}\right)\right\}^{2(r-1)}-1\right] .
$$

In fact, a simple computation shows that

$$
\begin{aligned}
\alpha & =A^{r\left(p-2 a^{\prime}-n+2 b^{\prime}\right)}\left[-\left(A^{2}+A^{-2}\right)\right]^{\mu(\gamma)-1} \\
& =A^{r\left(p-2 a^{\prime}-n+2 b^{\prime}\right)}\left[-\left(A^{2}+A^{-2}\right)\right]^{r\left(\mu\left(\gamma_{*}\right)-1\right)}\left[-\left(A^{2}+A^{-2}\right)\right]^{-2 k(r-1)} \\
& =\left\{A^{p-2 a^{\prime}-n+2 b^{\prime}}\left[-\left(A^{2}+A^{-2}\right)\right]^{\mu\left(\gamma_{*}\right)-1}\right\}^{r}\left[-\left(A^{2}+A^{-2}\right)\right]^{-2 k(r-1)} \\
& =\alpha_{*}^{r}\left[-\left(A^{2}+A^{-2}\right)\right]^{-2 k(r-1)} \\
& \equiv \alpha_{*}^{r} \bmod \left(\left[-\left(A^{2}+A^{-2}\right)\right]^{2(r-1)}-1\right) .
\end{aligned}
$$

Case B. $1 \mathrm{k}(L, F) \equiv 0(\bmod 2)$. 
We consider connected components of $\gamma \in S(a, b)$. Let $D_{0}, D_{1}, \ldots, D_{k}, \quad D_{\infty}, D_{1,1}, \ldots, D_{1, r}, D_{2,1}, \ldots, D_{2, r}, \ldots, D_{m, 1}, \ldots, D_{m, r}$, be connected components of $\gamma$ which satisfy (3.4) (2) and (3). Furthermore, $D_{0}$ and $D_{\infty}$ are such that

$$
\begin{aligned}
& D_{0} \text { contains }\{0\} \text { and } D_{\infty} \text { contains }\{\infty\} \text {, and } \zeta\left(D_{0}\right)=D_{0} \\
& \text { and } \zeta\left(D_{\infty}\right)=D_{\infty} \text {. }
\end{aligned}
$$

It may occur that $D_{0}=D_{\infty}$. We should note that $\gamma$ is the $r$-fold cyclic covering of $\gamma_{*}$ branched at 0 and $\infty$.

Now (3.5) (2) and (3) are still valid under the present case. Only (3.5) (1) should be changed to the following.

$$
\begin{aligned}
& \text { (i) If } D_{0} \neq D_{\infty} \text {, then } b_{1}\left(D_{0}\right)=r b_{1}\left(D_{0}^{\prime}\right) \text { and } \\
& b_{1}\left(D_{\infty}\right)=r b_{1}\left(D_{\infty}^{\prime}\right) . \\
& \text { (ii) } \text { If } D_{0}=D_{\infty} \text {, then } b_{1}\left(D_{0}\right)+1=r\left\{b_{1}\left(D_{0}^{\prime}\right)+1\right\} .
\end{aligned}
$$

Proof. (i) follows from the fact that $D_{0}$ and $D_{\infty}$ are, respectively, the $r$-fold cyclic coverings of $D_{0}^{\prime}$ and $D_{\infty}^{\prime}$ branched at 0 and $\infty$.

(ii) $D_{0}\left(=D_{\infty}\right)$ is the $r$-fold cyclic covering of $D_{0}^{\prime}$ branched at 0 and $\infty$. Let $d^{\prime}$ and $e^{\prime}$ denote the number of vertices and edges of $D_{0}^{\prime}$. Then

$$
\begin{aligned}
1-b_{1}\left(D_{0}\right) & =2+r\left(d^{\prime}-2\right)-r e^{\prime}=r\left(d^{\prime}-e^{\prime}\right)-2 r+2 \\
& =r\left(1-b_{1}\left(D_{0}^{\prime}\right)\right)-2 r+2,
\end{aligned}
$$

which yields $b_{1}\left(D_{0}\right)+1=r\left\{b_{1}\left(D_{0}^{\prime}\right)+1\right\}$.

Using (3.8) (i) and (3.5) (1), (2), we obtain the following formulas.

(i) When $D_{0} \neq D_{\infty}$,

$$
\begin{aligned}
\mu(\gamma)-1= & b_{1}(\gamma)+b_{0}(\gamma)-1 \\
= & b_{1}\left(D_{0}\right)+\sum_{i=1}^{k} b_{1}\left(D_{l}\right)+b_{1}\left(D_{\infty}\right) \\
& +\sum_{j=1}^{m} \sum_{\lambda=1}^{r} b_{1}\left(D_{j, \lambda}\right)+k+2+r m-1 \\
= & r b_{1}\left(D_{0}^{\prime}\right)+\sum_{i=1}^{k}\left\{r b_{1}\left(D_{i}^{\prime}\right)-r+1\right\} \\
& +r b_{1}\left(D_{\infty}^{\prime}\right)+\sum_{j=1}^{m} r b_{1}\left(D_{j, 1}^{\prime}\right)+k+1+r m
\end{aligned}
$$


(continued)

$$
\begin{aligned}
& =r\left\{b_{1}\left(D_{0}^{\prime}\right)+\sum_{i=1}^{k} b_{1}\left(D_{i}^{\prime}\right)+b_{1}\left(D_{\infty}^{\prime}\right)+\sum_{j=1}^{m} b_{1}\left(D_{j, 1}^{\prime}\right)+k+2+m-1\right\} \\
& -k r+k-r k-r-r m+k+1+r m \\
& =r\left\{\mu\left(\gamma_{*}\right)-1\right\}-(2 k+1)(r-1) . \\
& \quad \text { (ii) When } D_{0}=D_{\infty}, \\
& \begin{aligned}
\mu(\gamma)-1= & b_{1}\left(D_{0}\right)+\sum_{i=1}^{k} b_{i}\left(D_{i}\right)+\sum_{j=1}^{m} \sum_{\lambda=1}^{r} b_{1}\left(D_{J, \lambda}\right)+k+1+r m-1 \\
= & r b_{1}\left(D_{0}^{\prime}\right)+r-1+\sum_{i=1}^{k} r b_{1}\left(D_{i}^{\prime}\right)+\sum_{j=1}^{m} r b_{1}\left(D_{j, 1}^{\prime}\right)+k+r m \\
= & r\left\{b_{1}\left(D_{0}^{\prime}\right)+\sum_{i=1}^{k} b_{1}\left(D_{i}^{\prime}\right)+\sum_{j=1}^{m} b_{1}\left(D_{j, 1}^{\prime}\right)+k+1+m-1\right\} \\
& \quad-r k-r m+r-1+k+r m \\
= & r\left[\mu\left(\gamma_{*}\right)-1\right]-(k-1)(r-1) .
\end{aligned}
\end{aligned}
$$

Therefore, we have

$$
\alpha \equiv \alpha_{*}^{r} \bmod \left(\left[-\left(A^{2}+A^{-2}\right)\right]^{r-1}-1\right) .
$$

Now it only remains to show the following simple lemma.

LEMMA 6. For any prime $r$,

$$
\begin{aligned}
& (t+1)^{2(r-1)}-t^{r-1} \equiv \eta_{r}(t) \quad(\bmod r) . \\
& (t+1)^{r-1}-t^{r-1 / 2} \equiv \xi_{r}(t) \quad(\bmod r) .
\end{aligned}
$$

Proof. If $r=2$, the lemma is obvious. Therefore, we assume that $r$ is an odd prime. Then it suffices to prove the following.

(3.10) For $j=0,1, \ldots, r-1$,

$$
\begin{aligned}
& \text { (1) }\left(\begin{array}{c}
2 r-2 \\
j
\end{array}\right) \equiv(-1)^{j}(j+1) \quad(\bmod r), \\
& \text { (2) }\left(\begin{array}{c}
2 r-2 \\
r+j
\end{array}\right) \equiv(-1)^{j}(j+1) \quad(\bmod r), \\
& \text { (3) }\left(\begin{array}{c}
r-1 \\
j
\end{array}\right) \equiv(-1)^{j} \quad(\bmod r) .
\end{aligned}
$$


Proof. Firstly, (3) is obviously true for $j=0$ and 1. Since $\left(\begin{array}{c}r \\ j\end{array}\right)=\left(\begin{array}{c}r-1 \\ j\end{array}\right)$ $+\left(\begin{array}{c}r-1 \\ j-1\end{array}\right)$, it follows by the induction hypothesis that $0 \equiv\left(\begin{array}{c}r-1 \\ j\end{array}\right)+(-1)^{j-1}$ $(\bmod r)$ which yields $\left(\begin{array}{c}r-1 \\ j\end{array}\right) \equiv(-1)^{j}(\bmod r)$. This proves (3). Secondly, (1) is trivially true for $j=0$ and 1 . Now for $1 \leq j \leq r-1$,

$$
\left(\begin{array}{c}
2 r-2 \\
j
\end{array}\right)=\left(\begin{array}{c}
2 r-2 \\
j-1
\end{array}\right) \frac{2 r-j-1}{j} .
$$

Using the induction hypothesis, we can write

$$
\left(\begin{array}{c}
2 r-2 \\
j-1
\end{array}\right)=(-1)^{j-1} j+r k
$$

for some integer $k$. Then

$$
\left(\begin{array}{c}
2 r-2 \\
j
\end{array}\right)=(-1)^{j}(j+1)+(-1)^{j-1} 2 r+\frac{r k}{j}(2 r-j-1) .
$$

Since $\left({ }_{j}^{2 r-2}\right)$ is an integer and $r$ is a prime, $j \mid k(2 r-j-1)$ and hence

$$
\left(\begin{array}{c}
2 r-2 \\
j
\end{array}\right) \equiv(-1)^{j}(j+1) \quad(\bmod r) .
$$

This proves (1). Finally, since $r$ is odd and $r-j-2 \leq r-1$ for $0 \leq j \leq$ $r-1$, (3.10) (1) implies that

$$
\begin{aligned}
\left(\begin{array}{c}
2 r-2 \\
r+j
\end{array}\right) & =\left(\begin{array}{c}
2 r-2 \\
r-j-2
\end{array}\right) \equiv(-1)^{r-\jmath+2}(r-j-2+1) \\
& \equiv(-1)^{r-\jmath+1}(j+1) \equiv(-1)^{j}(j+1) \quad(\bmod r) .
\end{aligned}
$$

This proves (2).

Let $I$ be the ideal in $Z\left[A, A^{-1}\right]$ generated by $r$ and

$$
\left[-\left(A^{2}+A^{-2}\right)\right]^{2(r-1)}-1 \quad\left(\text { or }\left[-\left(A^{2}+A^{-2}\right)\right]^{r-1}-1 \text { in } Z\left[A, A^{-1}\right]\right)
$$

when $\operatorname{lk}(L, F) \equiv 1(\bmod 2)(\operatorname{or} \operatorname{lk}(L, F) \equiv 0(\bmod 2))$. The Lemma 6 yields that $P_{\tilde{L}}(A) \equiv\left[P_{\tilde{L}_{*}}(A)\right]^{r}(\bmod I)$. Let $w(\tilde{L})$ be the twisting number (or the writhe) of $\tilde{L}$. Then, since $w(\tilde{L})=r w\left(\tilde{L}_{*}\right)$, it follows that

$$
\begin{aligned}
f_{L}(A) & =(-A)^{-3 w(\tilde{L})} P_{\tilde{L}}(A)=(-A)^{-3 r w\left(\tilde{L}_{*}\right)} P_{\tilde{L}}(A) \equiv\left[(-A)^{-3 w\left(\tilde{L}_{*}\right)} P_{\tilde{L}_{*}}(A)\right]^{r} \\
& =\left[f_{L_{*}}(A)\right]^{r} \quad(\bmod I) .
\end{aligned}
$$

Here $f_{L}\left(t^{-1 / 4}\right)=V_{L}(t)[\mathbf{K}]$ and Theorem 1 follows from Lemma 6. A proof of Theorem 2 is now complete. 
4. Applications and remarks. Formula (1.1) may not be used to determine whether a knot (but not a link) $K$ has small prime period $r \leq 5$. In fact, we have the following

Proposition 7. Let $K$ be a knot. Then for $r=2,3$ or 5 ,

$$
V_{K}(t) \equiv 1 \bmod \left(r, \xi_{r}(t)\right) \text {. }
$$

Proof. First, note that $\xi_{2}(t)=1-t-\sqrt{t}, \xi_{3}(t)=1-2 t+t^{2}$ and $\xi_{5}(t)=1-t-t^{3}+t^{4}$. Now, as is well known (Definition $\left.17[\mathbf{J}]\right), 1-$ $V_{k}(t) \equiv 0 \bmod \xi_{5}(t)$, and hence $V_{K}(t) \equiv 1 \bmod \xi_{5}(t)$. Furthermore, congruences $1-t+t^{2} \equiv(1-t-\sqrt{t})(1-t-\sqrt{t})(\bmod 2),(1-t)\left(1-t^{3}\right)$ $\equiv\left(1-t+t^{2}\right)\left(1+t^{2}\right)(\bmod 2)$ and $(1-t)\left(1-t^{3}\right) \equiv\left(1-2 t+t^{2}\right)$. $\left(1+t+t^{2}\right)(\bmod 3)$ prove Proposition 7 .

It is also easy to show that for any prime $r \geq 5, \xi_{5}(t) \mid \xi_{r}(t)$.

Proposition 8. Let $r$ be an odd prime $\geq 5$. Let $\omega$ and $\tau$ denote, respectively, a primitive $(r-1) / 2$ th-root and $(r+1) / 2$ th-root of unity. If a link $L$ has period $r$, then

$$
\begin{aligned}
& V_{L}(\omega) \equiv V_{L_{*}}(\omega) \quad(\bmod r) \\
& V_{L}(\tau) \equiv V_{L_{*}}\left(\tau^{-1}\right) \quad(\bmod r) .
\end{aligned}
$$

Proof. From Theorem 1, we see that $V_{L}(t) \equiv V_{L_{*}}(t)^{r} \equiv V_{L_{*}}\left(t^{r}\right)$ $\bmod \left(r, \xi_{r}(t)\right)$. Note that

$$
\xi_{r}(t)=\frac{1+t^{r}}{1+t}-t^{(r-1) / 2}=\frac{1}{1+t}\left(1-t^{(r-1) / 2}\right)\left(1-t^{(r+1) / 2}\right) .
$$

Since

$$
\omega^{r}=\left(\omega^{(r-1) / 2}\right)^{2} \omega=\omega \text { and } \tau^{r}=\left(\tau^{(r+1) / 2}\right)^{2} \tau^{-1}=\tau^{-1},
$$

a substitution $\omega$ or $\tau$ for $t$ in $V_{L}(t)$ and $V_{L_{*}}\left(t^{r}\right)$ proves (4.2).

COROllary 9. Under the conditions of Proposition 8, if $L_{*}$ is unknotted, then

$$
V_{L}(\omega) \equiv V_{L}(\tau) \equiv 1 \quad(\bmod r)
$$

Using Corollary 9, we can prove the following

Proposition 10. The knot $10_{105}$ in $[\mathbf{R}]$ has no period. 
Proof. According to [B-Z, p. 312], 7 is the only possible period of $10_{105}$. Suppose that $K$ has period 7. Since $K$ is alternating and fibred [M1], the factor knot $K_{*}$ is either unknotted or fibred [M3]. Since $\Delta_{K}(t)=1-8 t+22 t^{2}-29 t^{3}+22 t^{4}-8 t^{5}+t^{6} \equiv(1+t)^{6}(\bmod 7)$, it follows from [M2] that $K_{*}$ must be unknotted. Therefore, by Corollary 9 , $V_{K}(\omega) \equiv 1$ and $V_{K}(\tau) \equiv 1(\bmod 7)$, where $\omega=e^{2 \pi i / 3}$ and $\tau=e^{2 \pi i / 4}$ $=\sqrt{-1}$. Since $V_{K}(t)=t^{-7}-4 t^{-6}+8 t^{-5}-12 t^{-4}+15 t^{-3}-15 t^{-2}+$ $14 t^{-1}-11+7 t-3 t^{2}+t^{3}$, we have $V_{K}(\sqrt{-1}) \equiv-1(\bmod 7)$. Therefore, $K$ cannot have period 7 .

REMARK. A similar argument reveals that if $K=10_{101}$ in $[\mathbf{R}]$ has period 7, then the factor knot cannot be unknotted.

\section{REFERENCES}

[B-Z] G. Burde-H. Zieschang, Knots, Walter de Gruyter (1985).

[J] V. F. R. Jones, A polynomial invariant for knots via von Neumann algebras, Bull. Amer. Math. Soc., 89 (1985), 103-111.

[K] L. H. Kauffman, State models and the Jones polynomial, (to appear).

[M1] K. Murasugi, On a certain subgroup of the group of an alternating link, Amer. J. Math., 85 (1963), 544-550.

[M2] _ On periodic knots, Comment. Math. Helv., 46 (1971), 162-174.

[M3] _ On symmetries of knots, Tsukuba J. Math., 4 (1980), 331-347.

[M4] Jones polynomials and classical conjectures in knot theory, Topology, 26 (1987), 187-194.

[R] D. Rolfsen, Knots and links, Publish or Perish Inc., (1976).

Received October 20, 1986 and in revised form December 1, 1986. This research was partially supported by NSERC No. A4034. 



\section{PACIFIC JOURNAL OF MATHEMATICS EDITORS}

\author{
V. S. VARADARAJAN \\ (Managing Editor) \\ University of California \\ Los Angeles, CA 90024 \\ HERBERT ClEMENS \\ University of Utah \\ Salt Lake City, UT 84112 \\ R. FINN \\ Stanford University \\ Stanford, CA 94305
}

\author{
HERMANN FLASCHKA \\ University of Arizona \\ Tucson, AZ 85721
}

RAMESh A. GANGolli

University of Washington Seattle, WA 98195

VAUGHAN F. R. JONES

University of California

Berkeley, CA 94720

\author{
ROBION KIRBY \\ University of California \\ Berkeley, CA 94720
}

C. C. MOORE

University of California

Berkeley, CA 94720

HAROLD STARK

University of California, San Diego

La Jolla, CA 92093

\section{ASSOCIATE EDITORS}
R. AREnS
E. F. BECKENBACH
B. H. NEUMANN
F. WOLF
K. YOSHIDA

(1906-1982)

\section{SUPPORTING INSTITUTIONS}

\begin{abstract}
UNIVERSITY OF ARIZONA
UNIVERSITY OF BRITISH COLUMBIA

CALIFORNIA INSTITUTE OF TECHNOLOGY

UNIVERSITY OF CALIFORNIA

MONTANA STATE UNIVERSITY

UNIVERSITY OF NEVADA, RENO

NEW MEXICO STATE UNIVERSITY

OREGON STATE UNIVERSITY
\end{abstract}

\author{
UNIVERSITY OF OREGON \\ UNIVERSITY OF SOUTHERN CALIFORNIA \\ STANFORD UNIVERSITY \\ UNIVERSITY OF HAWAII \\ UNIVERSITY OF TOKYO \\ UNIVERSITY OF UTAH \\ WASHINGTON STATE UNIVERSITY \\ UNIVERSITY OF WASHINGTON
}

The Supporting Institutions listed above contribute to the cost of publication of this Journal, but they are not owners or publishers and have no responsibility for its content or policies.

Mathematical papers intended for publication in the Pacific Journal of Mathematics should be in typed form or offset-reproduced (not dittoed), double spaced with large margins. Please do not use built up fractions in the text of the manuscript. However, you may use them in the displayed equations. Underline Greek letters in red, German in green, and script in blue. The first paragraph must be capable of being used separately as a synopsis of the entire paper. In particular it should contain no bibliographic references. Please propose a heading for the odd numbered pages of less than 35 characters. Manuscripts, in triplicate, may be sent to any one of the editors. Please classify according to the scheme of Math. Reviews, Index to Vol. 39. Supply name and address of author to whom proofs should be sent. All other communications should be addressed to the managing editor, or Elaine Barth, University of California, Los Angeles, California 90024.

There are page-charges associated with articles appearing in the Pacific Journal of Mathematics. These charges are expected to be paid by the author's University, Government Agency or Company. If the author or authors do not have access to such Institutional support these charges are waived. Single authors will receive $\mathbf{5 0}$ free reprints; joint authors will receive a total of $\mathbf{1 0 0}$ free reprints. Additional copies may be obtained at cost in multiples of 50 .

The Pacific Journal of Mathematics is issued monthly as of January 1966. Regular subscription rate: $\$ 190.00$ a year (5 Vols., 10 issues). Special rate: $\$ 95.00$ a year to individual members of supporting institutions.

Subscriptions, orders for numbers issued in the last three calendar years, and changes of address should be sent to Pacific Journal of Mathematics, P.O. Box 969, Carmel Valley, CA 93924, U.S.A. Old back numbers obtainable from Kraus Periodicals Co., Route 100, Millwood, NY 10546.

The Pacific Journal of Mathematics at P.O. Box 969, Carmel Valley, CA 93924 (ISSN 0030-8730) publishes 5 volumes per year. Application to mail at Second-class postage rates is pending at Carmel Valley, California, and additional mailing offices. Postmaster: send address changes to Pacific Journal of Mathematics, P.O. Box 969, Carmel Valley, CA 93924.

PUBLISHED BY PACIFIC JOURNAL OF MATHEMATICS, A NON-PROFIT CORPORATION Copyright (C) 1988 by Pacific Journal of Mathematics 


\section{Pacific Journal of Mathematics}

\section{Vol. 131, No. 2 December, 1988}

Selman Akbulut and Henry Churchill King, Polynomial equations of immersed surfaces .................................... 209

Alberto Baider and Richard C. Churchill, The Campbell-Hausdorff group and a polar decomposition of graded algebra automorphisms ........2 219

Wayne C. Bell and John William Hagood, Separation properties and exact

Radon-Nikodým derivatives for bounded finitely additive measures . . . 237

Dennis J. Garity, James P. Henderson and David G. Wright, Menger

spaces and inverse limits ...............................249

B. Brent Gordon, Algebraically defined subspaces in the cohomology of a

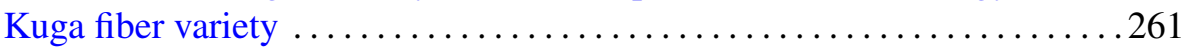

Jeffrey A. Hogan, Weighted norm inequalities for the Fourier transform on connected locally compact groups ........................... 277

Guojun Liao, A study of regularity problem of harmonic maps ..........291

Chin-pi Lu, Modules satisfying ACC on a certain type of colons ......... 303

Kunio Murasugi, Jones polynomials of periodic links

Hans Schoutens, Approximation properties for some non-Noetherian local

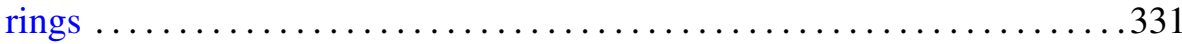

Peter Sjögren, Convergence for the square root of the Poisson kernel ...... 361 Alexandru Ion Suciu, The oriented homotopy type of spun 3-manifolds .393 